\title{
Supporting Information: Pool Boiling Coupled with Nanoscale Evaporation using Buried Nanochannels
}

\author{
An Zou, Sajag Poudel, Sidharth P. Raut, and Shalabh C. Maroo* \\ Department of Mechanical and Aerospace Engineering, Syracuse University, \\ Syracuse, NY 13244 \\ *Corresponding Author: scmaroo@syr.edu
}

\section{S1 Sample Fabrication}

The boiling samples were fabricated by etching a patterned sacrificing metal layer to form the cross-connected micro/nano channels. The shape of the channels is determined by the pattern of the sacrificing metal layers. Fig. S1-a shows the fabrication process for samples with channel height of $728 \mathrm{~nm}$ ( $\mathrm{S \#} \mathrm{1,} \mathrm{\# 3,} \mathrm{\# 4,} \mathrm{and} \mathrm{\# 5} \mathrm{in} \mathrm{main} \mathrm{manuscript).} \mathrm{The}$ fabrication started from a silicon ( $\mathrm{Si}$ ) wafer. $\sim 9 \mu \mathrm{m}$ thick photoresist was spinning coated on the wafer and then was exposed to the laser source, followed by an ammonia diffusion bake for the image reversal. After photoresist development, $713 \mathrm{~nm}$ thick copper $(\mathrm{Cu})$ was deposited using e-beam evaporator; $15 \mathrm{~nm}$ thick chromium (Cr) film was used as adhesion layer. Then, the photoresist was removed, together with the deposited $\mathrm{Cr} \& \mathrm{Cu}$ above it, by immersing the wafer in acetone for 6 hours. This lift-off process created cross-connected ridges of sacrificing $\mathrm{Cr} \& \mathrm{Cu}$, resulting in cross-connected channels as shown soon. Fig. $\mathrm{S} 2$ a shows a typical AFM image of the patterned sacrificing $\mathrm{Cr} \& \mathrm{Cu}$. A $300 \mathrm{~nm}$ thick $\mathrm{SiO}_{2}$ film were then deposited to bury the patterned $\mathrm{Cr} \& \mathrm{Cu}$ layer by plasma enhanced chemical vapor deposition (PECVD). A hole was fabricated by standard photolithography process, followed by dry etching of $\mathrm{SiO}_{2}$, above each intersect of $\mathrm{Cr} \& \mathrm{Cu}$ ridges. These holes expose the sacrificing $\mathrm{Cr} \& \mathrm{Cu}$ to the etchant in the next step and also connect the buried channels to bulk liquid in boiling experiments. At last, the wafer was immersed in $\mathrm{Cr}$ etchant, which also etches $\mathrm{Cu}$, to remove sacrificing $\mathrm{Cr} \& \mathrm{Cu}$ to form the buried channels. An AFM image of this cross-connected channels is shown in Fig. S2 b1. The fabrication of $\mathrm{S} \# 4$ is slight different as the bottom of the channel is $1 \mu \mathrm{m}$ thick PECVD $\mathrm{SiO}_{2}$, which was deposited before the coating of photoresist for lift-off process.

Fig. S1 b shows the fabrication process of $\mathrm{S} \# 2$ with channel height of $100 \mathrm{~nm}$. The difference here is the method to pattern the sacrificing metal layer. First, a $100 \mathrm{~nm}$ thick 
(a)

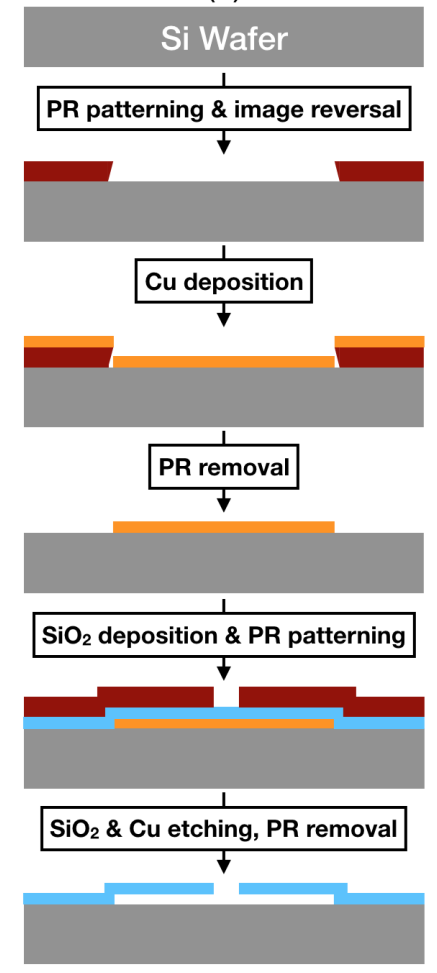

(b)

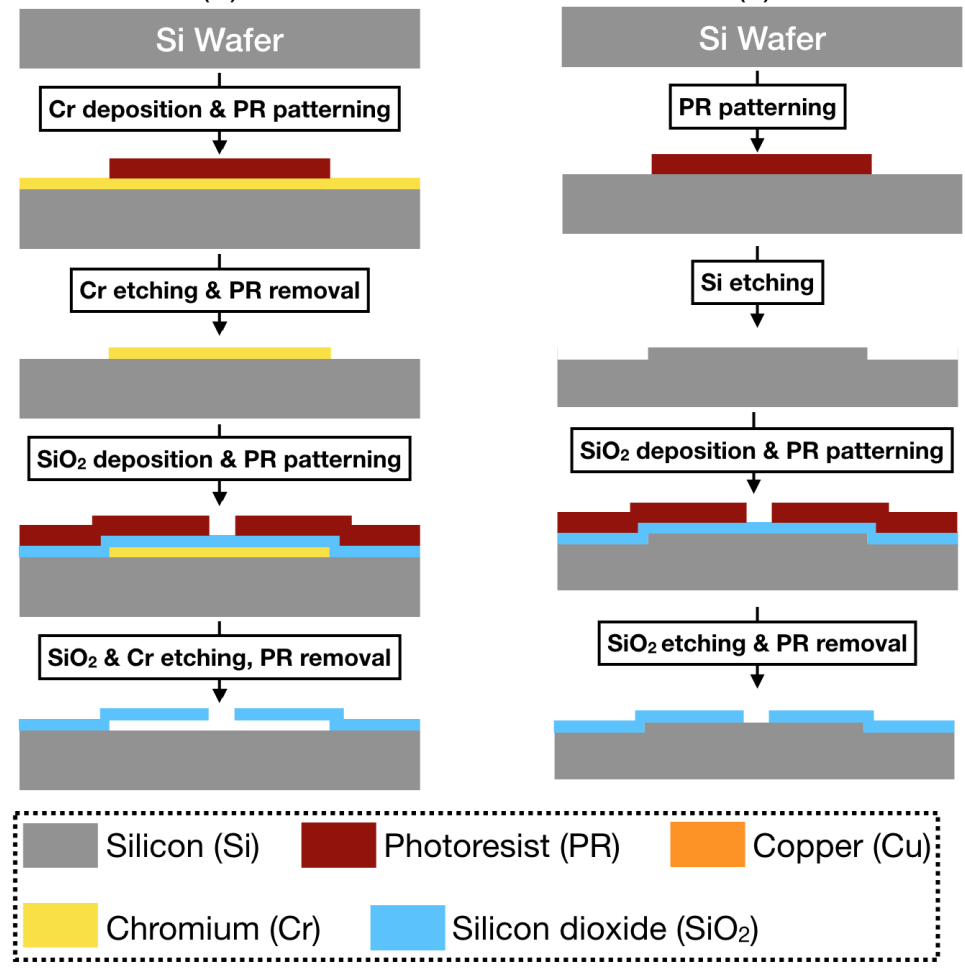

Figure S1: Fabrication process for samples (a) channel height of $728 \mathrm{~nm}(\mathrm{~S} \# 1, \# 3$, \#4, and \#5 in main manuscript), (b) channel height of $100 \mathrm{~nm}$ (S\# 2); and (c) sample with similar surface topology but without buried channels $(\mathrm{S} \# 6)$.

Cr layer was deposited by e-beam evaporation. Then $\sim 3 \mu m$ thick photoresist was spinning coated above this layer and patterned by standard photolithography process, followed by wet etching of $\mathrm{Cr}$. In this way, the sacrificing $\mathrm{Cr}$ was patterned as cross-connected ridges. After removing the remaining photoresist, a $300 \mathrm{~nm}$ thick PECVD $\mathrm{SiO}_{2}$ film was deposited to cover the sacrificing $\mathrm{Cr}$ layer. Following the same process as mentioned above, holes were fabricated to expose the sacrificing Cr layer to etchant, and last, the buried channels were formed by etching sacrificing Cr layer.

Fig. S1 c shows the fabrication process of $\mathrm{S} \# 6$, which has similar surface topology without buried channels. Cross-connected ridges were firstly fabricated by standard photolithography, following by dry etching of $\mathrm{Si}(796 \mathrm{~nm})$. Then $300 \mathrm{~nm} \mathrm{PECVD} \mathrm{SiO}_{2}$ was deposited and holes were fabricated at each ridge intersect to maintain the same surface topology as buried channels. Fig. S2 b2 shows an AFM image of this sample.

Both apparent contact angle $\left(\theta_{\text {app }}\right)$ and receding contact angle $\left(\theta_{r}\right)$ of all sample were measured using a goniometer. The apparent contact angle was obtained using a $\sim 0.5$ 

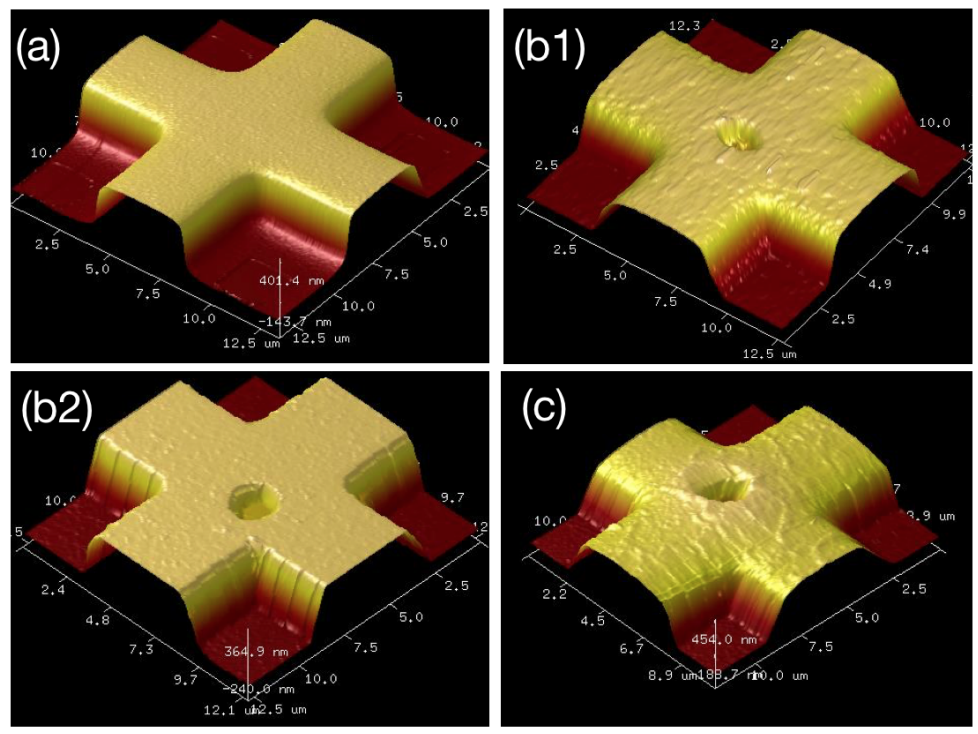

Figure S2: AFM images of (a) patterned sacrificing metal layer, (b1) sample with buried channels, (b2) sample has similar surface topology without buried channels, and (c) sample with buried channels after boiling experiments.

$\mu L$ water droplet sitting on the sample, while the receding contact angle was obtained by withdrawing liquid from a big drop sitting on the sample, and the snapshot were taken during the contact line retreat so that the image can be further analyzed. All measurements were repeated at least three times to ensure repeatability. Table S1 lists the results of all samples.

Table S1: Contact angles of tested samples

\begin{tabular}{lcc}
\hline Sample & $\theta_{a p p}\left(^{\circ}\right)$ & $\theta_{r}\left(^{\circ}\right)$ \\
\hline $\mathrm{S} \# 1$ & $15.0 \pm 1.6$ & $6.0 \pm 0.7$ \\
$\mathrm{~S} \# 2$ & $15.9 \pm 1.9$ & $6.2 \pm 0.7$ \\
$\mathrm{~S} \# 3$ & $10.7 \pm 1.2$ & $6.5 \pm 0.6$ \\
$\mathrm{~S} \# 4$ & $13.2 \pm 1.4$ & $5.9 \pm 0.8$ \\
$\mathrm{~S} \# 5$ & $14.3 \pm 1.4$ & $6.7 \pm 0.9$ \\
$\mathrm{~S} \# 6$ & $35.9 \pm 2.6$ & $19.0 \pm 2.1$ \\
\hline
\end{tabular}




\section{S2 Boiling Experiments}

The pool boiling experiment setup consists of a polycarbonate water bath and a thermal insulated copper block (Fig. S3). Five cartridge heaters are embedded in the copper block allowing for a maximum power input of $1250 \mathrm{~W}$ (heat flux of $1250 \mathrm{~W} / \mathrm{cm}^{2}$ ). The copper block is fixed inside an insulation housing made of PTFE Teflon (thermal conductivity, $k, \sim 0.25 \mathrm{~W} /(\mathrm{m} \cdot \mathrm{K}))$ and ceramic $(k<1 \mathrm{~W} /(\mathrm{m} \cdot \mathrm{K}))$. This heating assemble is further insulated by being surrounded by a 2 inch thick fiber glass sheet $(k \sim 0.042 \mathrm{~W} /(\mathrm{m} \cdot \mathrm{K}))$. The heat input to the copper block is controlled with a variable AC to AC transformer. Four equally spaced K-type thermocouples are inserted into the center axis of the copper block to measure the temperature gradient. In water bath, four immersion heaters and a RTD are used to maintain bulk water at its saturated temperature.

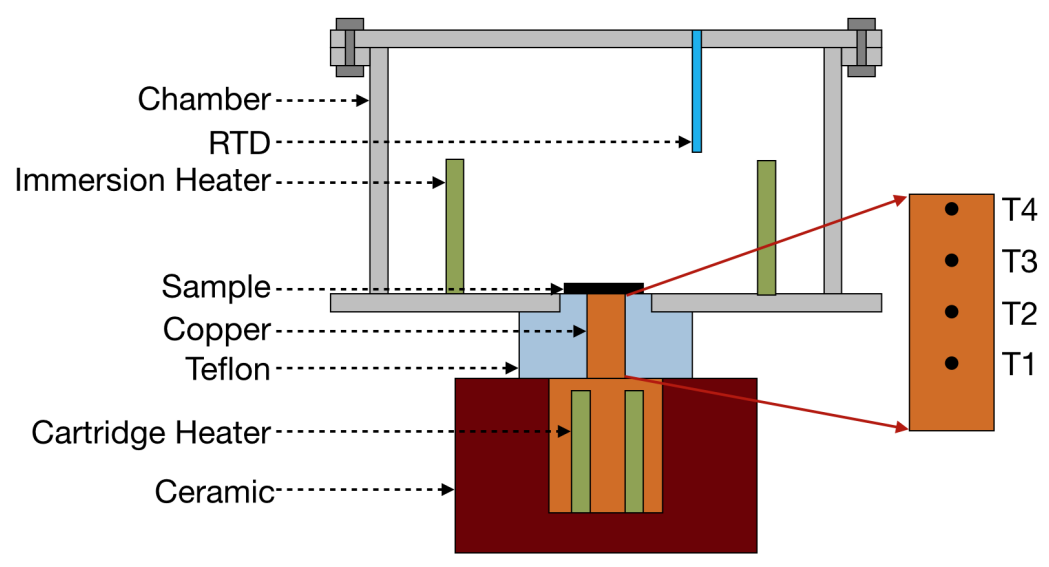

Figure S3: Boiling Setup

In boiling experiments, a typical sample is a $2 \times 2 \mathrm{~cm}$ square chip, with buried channels at the center of $1.4 \times 1.4 \mathrm{~cm}$. It was bonded on the top of the copper block using solder paste to ensure good attachment with minimal thermal contact resistance and the surface kept horizontally for all the experiments. The sample was immersed in a pool of deionized (DI) water, which was degassed by boiling it for $30 \mathrm{~min}$ and maintain it temperature at $97-100{ }^{\circ} \mathrm{C}$ for another $30 \mathrm{~min}$. Boiling on sample was achieved by increasing the output voltage of the variable transformer. To obtain the boiling curve, the output was increased in small increments. The temperature readings were recorded after reaching steady state, which is determined by the criterion that the temperature changes from all thermocouples were less than $0.5^{\circ} \mathrm{C}$ over $1 \mathrm{~min}$. The steady state was reached usually in $10-15$ min after changing the input power. CHF was obtained when an incremental increase of power supplied resulted in dramatic increase of surface temperature, and its value was taken as the last stable heat flux recorded during experiments. For each channel dimension, at least two samples were tested to ensure repeatability. The survival of the nanochannels after 
boiling experiments were confirmed by wicking test of a droplet (the same as shown in figure 4 in main manuscript) and AFM image (figure S2 c). The heat flux was obtained from measured temperature gradient using Fourier's law as Eq. S1.

$$
q^{\prime \prime}=-k_{C u} \cdot \frac{d T}{d x}
$$

where $k_{C u}$ is copper thermal conductivity. $\frac{d T}{d x}$ is obtained from the slope of the linear curve fitting of four temperature readings from thermocouples. $R^{2}$ value of the linear curve fitting is usually ranged from 0.9600 to 0.9990 when heat flux is lower than $15 \mathrm{~W} / \mathrm{cm}^{2}$, beyond which the $R^{2}$ value lies between 0.9993 and 0.9999 , implying good 1-D thermal conduction in the copper resulted from good thermal insulation. The surface temperature was obtained from the top thermocouple $\left(T_{4}\right)$, which is $762 \mu \mathrm{m}$ below the top surface of the copper, also using Fourier's law as Eq. S2. The temperature gradient across the solder film between copper and sample was ignored.

$$
T_{s}=T_{4}-q^{\prime \prime} \cdot\left(\frac{L_{C u}}{k_{C u}}+\frac{t_{S i}}{k_{S i}}\right)
$$

where $L_{C u}$ is the distance between the thermocouple and top of the copper; $t_{S i}$ and $k_{S i}$ are thickness and thermal conductivity of the Si wafer respectively.

The uncertainties of heat flux and surface temperature were propagated from temperature measurement, curve fitting, distance measurement, etc. The uncertainty of the temperature measurement of the thermocouple was $1.1 \mathrm{~K}$; while the uncertainty of thermocouple location was less than $0.1 \mathrm{~mm}$. The uncertainty of temperature gradient $\left(E_{d T / d x}\right)$ is the sum of error from these two measurement and the error from linear curve fitting. Eq. S3 and Eq. S4 were used for determining the uncertainty of heat flux and surface temperature respectively. The maximum uncertainties of heat flux and surface temperature are $4.05 \mathrm{~W} / \mathrm{cm}^{2}$ and $1.54{ }^{\circ} \mathrm{C}$ respectively in current work.

$$
\begin{gathered}
E_{q "}=k_{C u} \cdot E_{d T / d x} \\
E_{T_{s}}=\sqrt{\left(\frac{\partial T_{s}}{\partial T_{4}}\right)^{2}\left(E_{T_{4}}\right)^{2}+\left(\frac{\partial T_{s}}{\partial q^{\prime \prime}}\right)^{2}\left(E_{q "}\right)^{2}+\left(\frac{\partial T_{s}}{\partial L_{C u}}\right)^{2}\left(E_{L_{C u}}\right)^{2}}
\end{gathered}
$$

\section{S3 CHF-W Model and Surface Wickability Characterization}

To determine the role of wicking on our CHF enhancement, we compared our experimental data to a widely used CHF model which predicts CHF enhancement only due to the wicking capability of a surface (CHF-W model) [1]. CHF-W model can also be written in the form of Eq. S5 (same as Eq. 1 in main manuscript with different constant $K$ value).

$$
q_{C H F}=K_{W} \cdot \rho_{v}^{1 / 2} h_{l v}\left[\sigma_{l v} g\left(\rho_{l}-\rho_{v}\right)\right]^{1 / 4}
$$



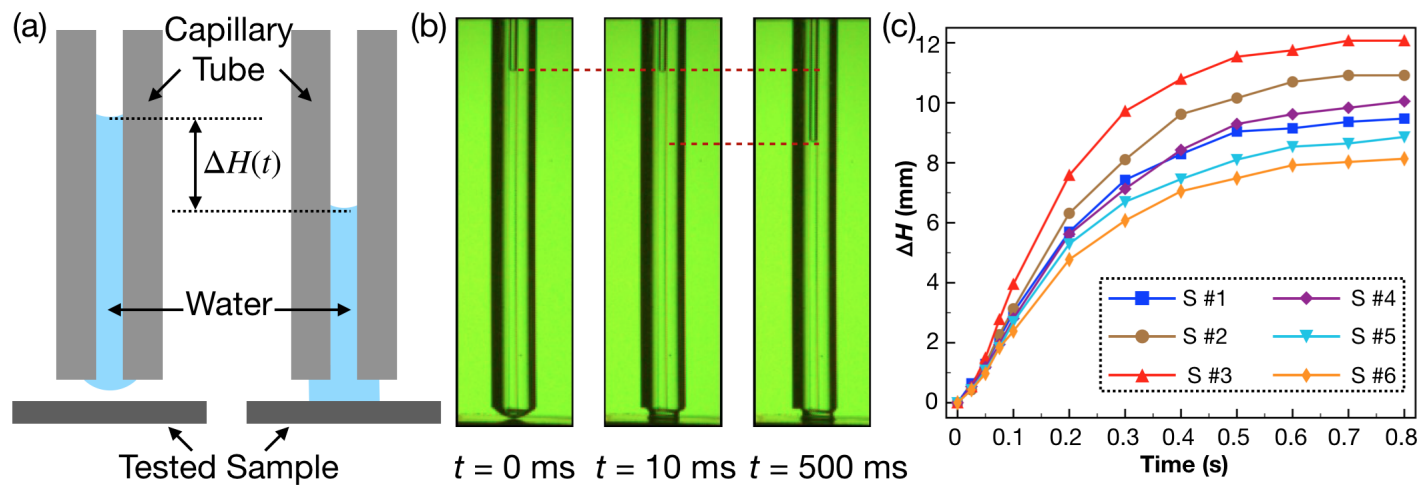

Figure S4: Surface wickability characterization. (a) sketch of wickability test; (b) high speed images of water column inside the tube after the tested surface touches the hanging droplet; (c) experimental results of changes of water column height with time.

where $K_{W}$ is a constant and its value can be determined from Eq. S6; $\rho_{l}$ and $\rho_{v}$ are liquid and vapor density respectively, $h_{l v}$ is latent heat, $\sigma_{l v}$ is liquid-vapor surface tension, and $g$ is gravitational constant.

$$
K_{W}=K_{Z} \cdot\left\{1+\frac{\dot{V}_{0}^{\prime \prime} \rho_{l}}{\rho_{v}^{1 / 2}\left[\sigma_{l v} g\left(\rho_{l}-\rho_{v}\right)\right]^{1 / 4}}\right\}
$$

where $K_{Z}$ is the constant $K$ in Zuber's hydrodynamic CHF model [2], and its value is 0.131 ; the second term in wavy bracket is the wicking number $W_{i}$, in which $\dot{V}_{0}^{\prime \prime}$ defines the surface wickability and it is determined as the initial wicked volume flux when the surface is in contact with pendant droplet.

The method to determine $\dot{V}_{0}^{\prime \prime}$ was adopted from literature $[1,3]$. As shown in Fig. S4 a, a glass capillary tube (inner diameter of $500 \mu \mathrm{m}$, outer diameter of $5.5 \mathrm{~mm}$ ) was first filled up with DI water to a height of $\sim 40 \mathrm{~mm}$ using a syringe pump. A pensile water droplet formed at the end of the tube. The tested surface was raised slowly using a micrometer translation stage until it touched the droplet. The water spread on the surface, causing the height of water in the tube to decrease. A high-speed camera was used to record the water height change with time $(\Delta H)$ during this spreading at 1000 frames per second (fps). It was observed that the water filled the gap between tube end and the surface within 10 $\mathrm{ms}$, reaching a stable wetted area with a diameter $\left(D_{W}\right)$ of $3.89-4.06 \mathrm{~mm}$. The water height in the tube was not observed any change when water filled the gap between tube and surface, it started to decrease when the water started to wick into the channels (Fig. S4 b). Fig. S4 c shows plots of $\Delta H$ versus time for all samples tested in boiling experiments.

With measured $D_{W}$ and known tube inner diameter $\left(D_{t}\right)$, the initial wicked volume flux when the surface is in contact with the droplet can be determined by Eq. S7.

$$
\dot{V}_{0}^{\prime \prime}=\left.\frac{1}{A_{W}}\left(\frac{d V}{d t}\right)\right|_{t=0}=\left.\left(\frac{D_{t}}{D_{W}}\right)^{2}\left(\frac{d H}{d t}\right)\right|_{t=0}
$$



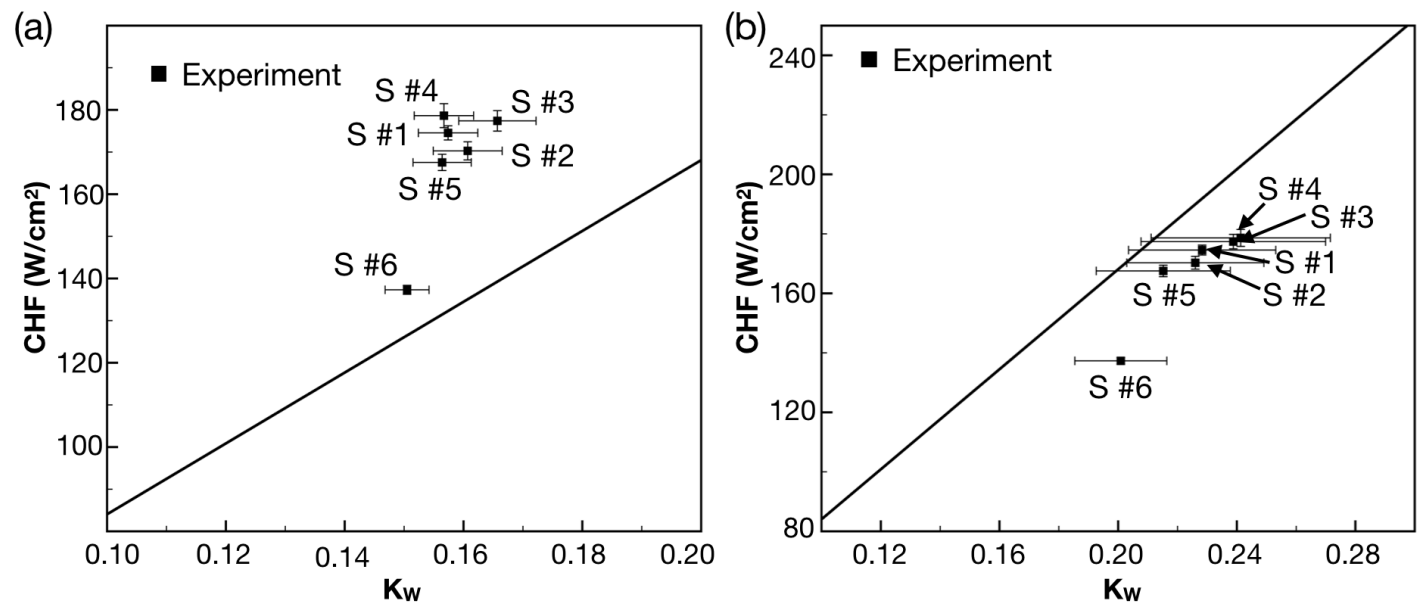

Figure S5: Comparison of experimental data to CHF-W model using tube of (a) 500 $\mu \mathrm{m}$ inner diameter $5.5 \mathrm{~mm}$ outer diameter and (b) $400 \mu \mathrm{m}$ inner diameter $800 \mu \mathrm{m}$ outer diameter.

where $A_{W}$ is wetted area; $V, H$ are the water volume and height in tube respectively.

To predict CHF, Eq. S6 was used to calculate the modified constant $K_{W}$. Fig. S5 a shows the comparison of experimental CHF values to the CHF-W model prediction. The model underestimated CHF by $27 \%$ - 32\% except for S \#6 (10\%). It was also found that the wicked volume flux was highly dependent on capillary tube dimensions as the diameter of the wetting area was affected by the tube wall thickness. Thus, the surface wickability was also tested with FDTS coated thin glass tube (inner diameter $400 \mu \mathrm{m}$, outer diameter $800 \mu \mathrm{m})$. Fig. S5 b shows comparison of experimental CHF valued to the CHF-W model prediction based on $K_{W}$ values measured with thin tube. The model overestimates by $7 \%$ - $12 \%$ except for S\# 6 (19\%).

\section{S4 CFD}

CFD simulations are performed on the same geometry as $\mathrm{S} \# 3$ using a commercial CFD solver ANSYS Fluent with multiphase volume of fluids (VOF) model. Uni-directional phase change due to evaporation at liquid-vapor interface was implemented where the interface was tracked based on volume fraction of liquid $~ 0.5$. Also, liquid-vapor phase interaction was simulated using Lee model which takes care of high rate of evaporation of liquid at $100{ }^{\circ} \mathrm{C}$ based on the saturation temperature. The surface tension effect is included by continuum surface force model along with wall adhesion. Fig. S6 shows the CFD simulation domain. To reduce the computational cost, a cylindrical droplet was used and only $1 / 8$ part of the system was included in the simulation with symmetrical boundary condition at the both side walls, and pressure outlet boundary (1 atm) at the top and outer 
walls. No-slip boundary condition was applied on substrate and all walls of the channels with an intrinsic contact angle of $30^{\circ}$. The temperature of the whole domain was set as 25 ${ }^{\circ} \mathrm{C}$ except the substrate and channel wall temperature, which was set as $25^{\circ} \mathrm{C}$ and $100^{\circ} \mathrm{C}$ respectively for two cases with different surface temperature $\left(T_{s}\right)$.

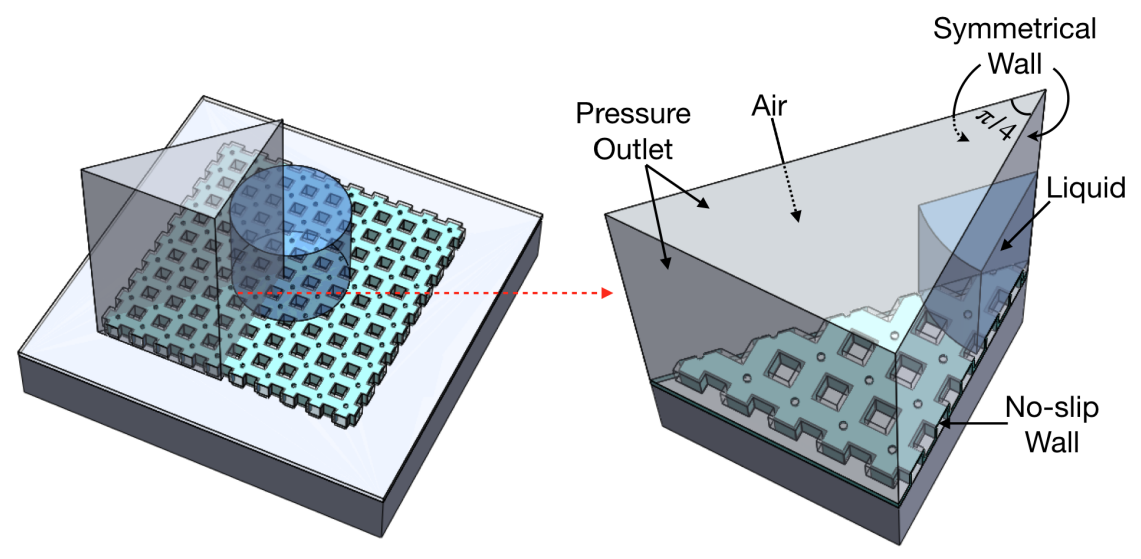

Figure S6: CFD simulation domain and boundary conditions

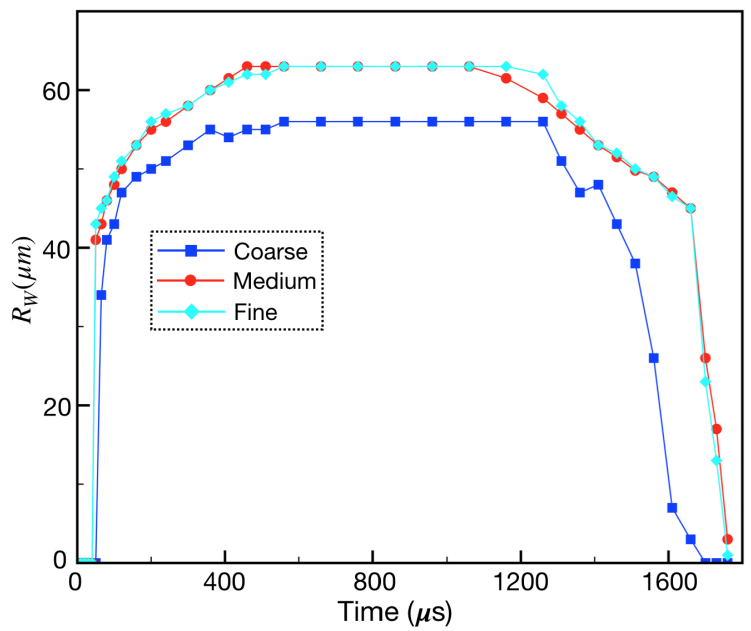

Figure S7: Grid independence study

Mesh was generated using ANSYS Workbench. Hexahedral dominant method is applied in the tank part of the computational domain whereas tetrahedral type is opted for constructing mesh on channel/pores part. Three different sizes of mesh were created: coarse mesh with 0.1 million cells in total, medium mesh with 0.2 million cells, and fine mesh with 0.5 million cells. The wicking radius $R_{W}$ was plotted versus time to test the 
grid independence (Fig. S7). The result from medium mesh was in good agreement to that from fine mesh, thus the medium mesh was used for the further simulations.

\section{References}

[1] M. M. Rahman, E. Ölçeroğlu, and M. McCarthy, "Role of wickability on the critical heat flux of structured superhydrophilic surfaces," Langmuir, vol. 30, no. 37, pp. 1122511234, 2014.

[2] N. Zuber, Hydrodynamic Aspects Of Boiling Heat Transfer (Thesis). University of California: Los Angeles, Cailfornia, 1959.

[3] H. S. Ahn, G. Park, J. M. Kim, J. Kim, and M. H. Kim, "The effect of water absorption on critical heat flux enhancement during pool boiling," Experimental Thermal and Fluid Science, vol. 42, pp. 187 - 195, 2012. 